\title{
Carrier ethernet network control plane based on the Next Generation Network
}

\author{
Fu, Rong; Wang, Yanmeng; Berger, Michael Stubert
}

Published in:

Proceedings of First ITU-T Kaleidoscope Academic Conference - Innovations in NGN: Future Network and Services 2008

Link to article, DOI:

10.1109/KINGN.2008.4542279

Publication date:

2008

Document Version

Publisher's PDF, also known as Version of record

Link back to DTU Orbit

Citation (APA):

Fu, R., Wang, Y., \& Berger, M. S. (2008). Carrier ethernet network control plane based on the Next Generation Network. In Proceedings of First ITU-T Kaleidoscope Academic Conference - Innovations in NGN: Future Network and Services 2008 (pp. 293-298). IEEE. https://doi.org/10.1109/KINGN.2008.4542279

\section{General rights}

Copyright and moral rights for the publications made accessible in the public portal are retained by the authors and/or other copyright owners and it is a condition of accessing publications that users recognise and abide by the legal requirements associated with these rights.

- Users may download and print one copy of any publication from the public portal for the purpose of private study or research.

- You may not further distribute the material or use it for any profit-making activity or commercial gain

- You may freely distribute the URL identifying the publication in the public portal 


\title{
CARRIER ETHERNET NETWORK CONTROL PLANE BASED ON THE NEXT GENERATION NETWORK
}

\author{
Rong Fu, Yanmeng Wang, Michael S.Berger \\ DTU Fotonik \\ Oersteds plads building 343 DK-2800 \\ Kgs. Lyngby \\ rfu@com.dtu.dk, yawa@cybercity.com,msb@com.dtu.dk
}

\begin{abstract}
This paper contributes on presenting a step towards the realization of Carrier Ethernet control plane based on the Next Generation Network (NGN). Specifically, Transport MPLS (T-MPLS) is taken as the transport technology in Carrier Ethernet.

It begins with providing an overview of the evolving architecture of the Next Generation Network (NGN). As an essential candidate among the NGN transport technologies, the definition of Carrier Ethernet (CE) is also introduced here.

The second part of this paper depicts the contribution on the T-MPLS based Carrier Ethernet network with control plane based on NGN architecture. The approaches to QoS mapping, label distribution and Connection and Admission Control (CAC) are specified here.

At last, a simple T-MPLS based Carrier Ethernet network model with three kinds of users (VoIP, VoD and HTTP) and a RACF based control module is simulated in OPNET. The model is aiming at illustrating the improvement of the Carrier Ethernet network with the NGN control plane.
\end{abstract} $\mathrm{CAC}$

Keywords - NGN, Carrier Ethernet, T-MPLS, QoS,

\section{INTRODUCTION}

In the old days, most of the network architectures were designed referring to the OSI 7-Layer reference model. Since it has the architecture division concept mainly on the vertical direction, the strict requirement on the "step by step enhancement [2]" in every 1-6 layers places big restriction on network service deployment, management and convergence. Moreover, another set of issues coming from the conventional network, which is also the main concern in this paper, is about the demanding of an intelligent network resource control and management system. Since there are some network technologies carried out (e.g. IP/MPLS) do have the control plane implemented on top of, but still the problems are not solved in principle. For instance in the IP/MPLS network, the control planes are

This work is supported in part by the Danish National Advanced Technology foundation project HIPT (High Quality IP network for IPTV and VoIP). implemented and distributed among the MPLS enabled devices in the whole network. When network resource reservation or information recollection takes place, the signaling and routing procedures (e.g. RSVP and OSPF) add extra workload on the network scalability. Moreover this sort of distributed control plane is not underlying transport technologies friendly. GMPLS (Generalized MPLS) and ASON (Automatically Switching Optical Network) are both on the way to propose their control systems unified and centralized. The philosophies in the GMPLS/ASON control plane are quite close to the NGN control plane. In the case of the control plane centralization, there is no need to use the OSPF procedures for the information collection thus the scalability problem seems solved. However, even in the centralized case, people still incline on inheriting the signaling procedure of RSVP (RSVP-TE). Therefore, the network resource reservation initialized by the users' side but not the control plane driven still seems not perfectly compliance with the next generation network spirits.

The Next Generation Network (NGN) framework carried out by ITU-T enrolled with a set of advanced features such as end-to-end quality assurance and intelligent network resource management system hit the hottest spot among the plenty of network constructing approaches. The newly and revolutionary characteristics in the NGN is aiming at overcoming the issues generated by the old or conventional network architecture and supplying an open, neutral and standardized environment to every network operators.

Together with the development of the network architecture the concept called "carrier class service" in the transport network services perspective is also getting popular. The Carrier Ethernet (CE) defined by Metro Ethernet Forum (MEF) is the pioneer in this area. Five attributes of the Carrier Ethernet makes itself an excellent candidate in the part of the NGN transport layer; and from the implementation of the Carrier Ethernet, the desires on a centrally managed, standards-based and vendor independent control plane requires the NGN architecture. To Carrier Ethernet, the control and transport functions separation and independence are the most valuable parts in the NGN. In the carrier class Ethernet, the transport functions are all located in the second layer while it formulates and integrates the control functions to a centralized independent control plane. Thus in most of the cases there is no need to do the dynamic routing decisions, 
which is a very attractive solution to the network scalability issues that bother the network developers a lot according to the old generation network architecture.

In reality, there are already investigations on the realization of the carrier class Ethernet and accompanied with network solutions claimed that they already achieved the technology independent control plane operation system. Nevertheless, the doubt is that without widely accepted and standardized references or recommendations, how far can this kind of solution go? And without such a unified control plane, the carrier class Ethernet could be confined with quite a few carriers instead of the general carriers.

\section{THE EVOLUTION OF THE NGN CONTROL PLANE}

The NGN framework proposed by ITU-T (recommendation Y.2001, Y.2011) brings in an evolutional functionality division methodology. The idea of the function division in the NGN is to keep the diversity of the existing network services and technologies while discomposes its architecture into horizontal and vertical two dimensions. The purpose is to declare the independent roles on network control and transport functions. The set of transport functions reside in NGN transport stratum are merely concerning with the packets conveyance and leave the control plane with all of the functions related to the network resource control and management.

In NGN, this control plane is visualized to the Resource and admission control functions (RACF) and Network attachment control functions (NACF). There are function blocks related to the service control (e.g. user authentication, user profile management, service admission control etc.) and function blocks taking the charges of the transport network(s) control (e.g. access admission control, network resource/policy control, dynamic connectivity provision etc.) as well. Differ from traditional control plane distribution scheme, one of the advanced aspects of this NGN control plane is the logically integrated and physically distributed functional components. Unlike the IP/MPLS distributing its control functions in every switch component in the transport layer, RACF gathers all the tasks about transport resource control into one system to keep the simplicity of the transport network. However, the features of unified, standardized and underlying technology agnostics of the NGN control plane make it suitable to all kinds of transport networks and the distributed control components keeps the data delivery seamless and flexible.

\section{CARRIER ETHERNET}

\subsection{Carrier Ethernet service attributes}

Attributed to its optimal fast data transfer technology, cost effective and simple infrastructure, the conventional Ethernet transport technology has been dominant in the enterprise LAN (Local Area Network) for many years. However, in the areas of MAN (Metro Area Network) and WAN (Wide Area Network), the Ethernet has not taken the leader role, instead the more expensive and complicated technologies such as ATM and Frame Relay still occupy these areas. With the more investigating on the quality assured and classified transport services and the concern on the infrastructure simplicity, the best effort, one-fits-all Ethernet transport obviously does not fulfill with these requirements. Thus Metro Ethernet Forum (MEF) defined the concept of Carrier Ethernet (CE). The Carrier Ethernet here is more general than a specific Ethernet transport technologies. It is more like an open defined concept including all the technologies and services which can achieve all common attributes for a carrier class Ethernet. In MEF, Carrier Ethernet is demanded by the five main attributes: [4] Standardized Services, Scalability, Reliability, Quality of service, Service of management independence.

This five attributes can also be taken as the main criterions in judging and valuing the transport technologies for the NGN.

\subsection{Carrier Ethernet transport technology -- T-MPLS}

Transport MPLS (T-MPLS) defined by ITU-T recommendations G.8110.1, G8112, G.8121 is a connection-oriented MPLS based packet transport technology.

The forwarding behavior of T-MPLS is a subset of MPLS, while abandons the control functions that implemented in the IP/MPLS network. It simplifies the data plane, removes unnecessary forwarding process such as PHP and label merging from the MPLS behaviors, and adds ITU-T transport style protection switching and OAM (Operation, Administration and Maintenance) functions. These simplifications and additions make T-MPLS a purely transport-oriented and "carrier-based" transport network technology. Moreover, different from the MPLS technology depending control functions, T-MPLS is meant to be independent of the network control plane, which provides wide network scalability and flexibility.

For the goal of traffic engineering and end-to-end transport, T-MPLS does not support connectionless transfer mode. All the connectivity it provides is explicit point to point connection. In the meantime T-MPLS removes the unnecessary label behaviors including:

- Using bi-directional LSPs (Label Switch Paths).

- $\quad$ No PHP (Penultimate Hop Pop)

- No LSP merging option

- $\quad$ No ECMP (Equal-Cost Multi-Path)

And in the case of metro area network, the label for every tunnel is normally configured by the management system, this feature saves the time on the label distributing processing and also makes the QoS mapping between the network control and transport network much easier.

\section{CONTROL PLANE FOR CARRIER ETHERNET BASED ON NGN ARCHITECTURE}

The resource and admission control functions (RACF) in NGN control plane is specified by ITU-T recommendation 
Y.2111. As described above, the transfer functions in the NGN transport layer just concern the data conveyance from one point to another, thus the RACF takes responsibilities of all the related admission and control functions. It can also be considered as a tie connecting the service and transport network, and transforming the information exchanged between them.

Fig 1 is a simple Carrier Ethernet solution with the control plane based on NGN. In the RACF block, two main function entities PD-FE (entities policy decision function entity) and TRC-FC (transport resource control function) perform the technology independent and technology dependent network resource control respectively. The PDFE provides a single contact point between the service stratum and the transport stratum. [3] It hides the underlying transport technologies for the service control functions (SCF) and it is technology independent as well. All the final policy decisions are made here with the consulting to the TRC-FE (Transport resource control function entity) for the technology dependent information such as the network resource availability held by the instances in TRC-FE. After making the decision, the policy is also pushed down to the accordingly policy enforcement function entity (PE-FE) in the transport network.

Considering the T-MPLS based Carrier Ethernet scenario, the functions executed inside the PD-FE will not make difference, however the TRC-FC has to keep a specific instance that aiming for this T-MPLS based transport network. According to the specific requirements of the TMPLS realized carrier class Ethernet transport network, the TRC-FC should perform the following functions:

- Transport network topology and link capacity maintenance.

- Call and admission control (CAC).

- Technology dependent QoS requirement and attributes mapping from service level to network level.

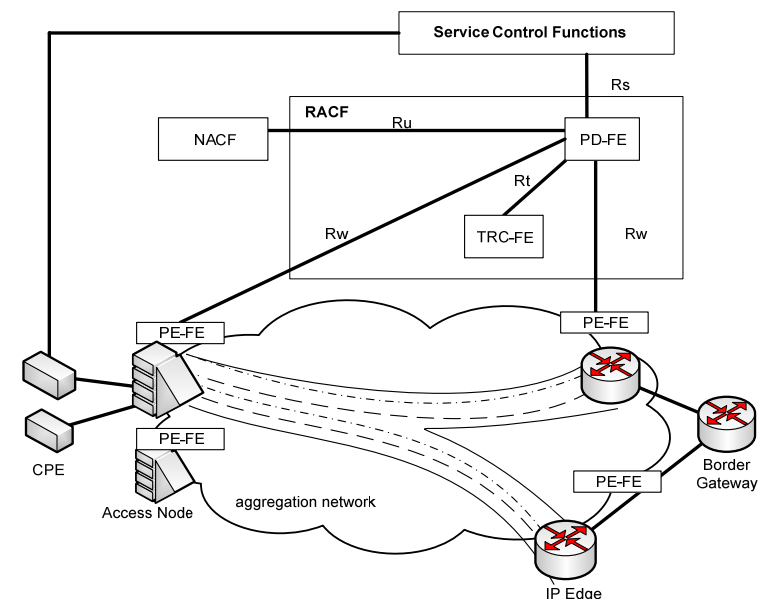

Fig. 1. Carrier Ethernet with control plane based on NGN

The policy enforcement function entities (PE-FE) residing in the edge devices at the T-MPLS network should perform the following functions:

- Flow based traffic policing and shaping.

- T-MPLS label distribution.

\subsection{Call and admission control in TRC-FE}

The call and admission control (CAC) function held in TRC-FE not only calls for the QoS mapping from the PDFE, but also the transport network topology and network link resource information maintenance. As common, most of the links in the transport network are conjunct along the path, and there probably will be more than one tunnels go through the link and reserve the bandwidth. The accurate capacity of each link in the transport network has to be recorded and kept up to time. Due to there could be different numbers of tunnels sharing the same links; the left capacity of each link will differ after a certain times of reservation. The algorithm introduced below suits for the Carrier Ethernet link layer resource information maintenance.

Taking the example of T-MPLS transport network in Fig. 2 , the T-MPLS enabled switches A, B, C and D are connected by Link 1, Link 2 and Link 3. Tunnel 1 goes through Link 1 and 3 while Tunnel 2 goes through Link 2 and Link 3. Before the network is switched on, all of the links are held the same capacity.

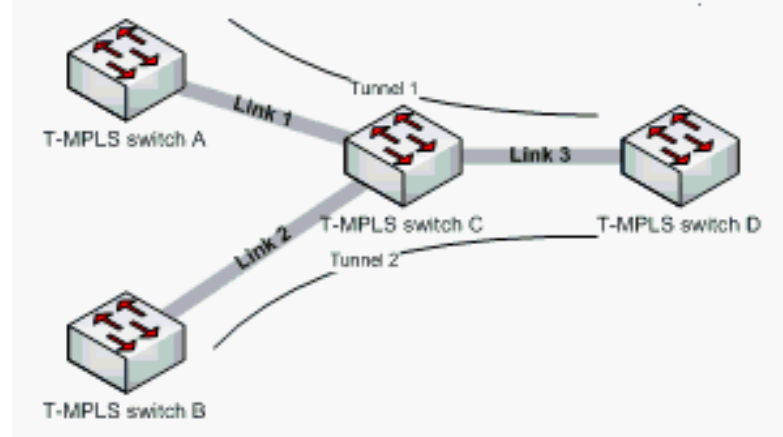

Fig. 2. T-MPLS transport network link

Before the network switched on, the TRC-FE instance for this transport network will inquire or be informed with the tunnel composition and the link capacity information. All of this information are maintained and updated in the database in TRC-FE. Table 1, Table 2 and Table 3 provide an example of the link layer information on the link capacity, tunnel composition and tunnels capacity respectively.

Table 1 Initial link capacity

\begin{tabular}{|c|c|}
\hline Link Number & Link Capacity $(\mathrm{M})$ \\
\hline 1 & 1000 \\
2 & 1000 \\
3 & 1000 \\
\hline
\end{tabular}

Table 2 Network topology

\begin{tabular}{|c|c|}
\hline Tunnel Number & Composed Links \\
\hline 1 & 1,3 \\
2 & 2,3 \\
\hline
\end{tabular}

Table 3 Tunnel capacity

\begin{tabular}{|c|c|}
\hline Tunnel Number & Tunnel Capacity (M) \\
\hline 1 & 1000 \\
2 & 1000 \\
\hline
\end{tabular}


As soon as the network is switched on, a network reservation on either the Tunnel 1 or Tunnel 2 will take place. Assuming there is a flow reservation request of 100 $\mathrm{M}$ on Tunnel 1. As it is the first request, the TRC-FE will first look into the network initialization information from Table 3, it shows that there is enough capacity for the required reservation on tunnel 1 , and the request can be granted. After this reservation confirmed, the reserved bandwidth will be eliminated from the link capacity table, a new table (Table 4) will be formed to record the left link capacity. In this table both the Link 1 and 3 has the left bandwidth of $900 \mathrm{M}$ while Link 2 is not part of Tunnel 1 remains unchanged. Accordingly, the table maintaining the tunnel capacity information should also be reformed and updated. For the Link 3 is the common link shared by both Tunnel 1 and Tunnel 2, and the tunnel bandwidth capacity here is just counted as the smallest link capacity along the tunnel. Thus, the tunnel capacities for both Tunnel 1 and 2 are decreased to $900 \mathrm{M}$. Next time when there is a new reservation request coming, the TRC-FE will go to the tables maintaining the latest link and tunnel capacity and check the network availability for granting the request.

Table 4 Link capacity after reservation

\begin{tabular}{|c|c|}
\hline Link Number & Link Capacity (M) \\
\hline 1 & 900 \\
2 & 1000 \\
3 & 900 \\
\hline
\end{tabular}

Table 5 Tunnel capacity after reservation

\begin{tabular}{|c|c|}
\hline Tunnel Number & Tunnel Capacity (M) \\
\hline 1 & 900 \\
2 & 900 \\
\hline
\end{tabular}

\subsection{Double label scheme in QoS traffic class mapping and policy pushing}

To achieve the QoS transport, the RACF in the control plane should map the QoS requirements from the application level to the transport level and push them as flow based policy down to the correspondent PE-FE (policy enforcement function entity). The transport network should refer to this policy information and perform the correspondent transfer behaviours to different traffic flows. This paper illustrates the schemes on the QoS maps taking place between the T-MPLS control and transfer functions. It also carried out a double label scheme on T-MPLS based transport network which using double labels to carry the information such as policy and service priority during packets transport.

The mapping between the SCF (service control functions) and the PD-FE (policy decision function entity) is underlying technology independent; there is no need to specify a scenario for Carrier Ethernet.

Upon receiving the request from the service layer, the PDFE will do the resource availability check by inquiring the related TRC-FE and try to install the policy into the correspondent underlying routers. To achieve the QoS transport and flow based policy in the T-MPLS transport layer, two identifiers are needed to associate with one traffic flow: the service/user ID and the flow ID. The former one will be treated as the indicator to both the LSP to the destination and the service class. As illustrated in Fig. 3, the thick grey tunnels are the LSPs designating to difference service classes. The service IDs are gained from the PD-FE according to the service level QoS requirements. The latter one can be used as the indicator for traffic policing at the edge routers. In Fig. 3, they stand for the thin tubes inside the LSPs.

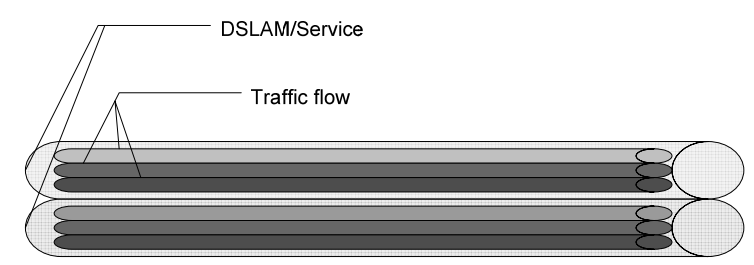

Fig.3. T-MPLS double label scheme

In the T-MPLS based transport network, these two IDs will be mapped into two labels in every packet, as shown in Fig. 4.

\begin{tabular}{|c|}
\hline Outer label \\
\hline Inner label \\
\hline Ethernet packet \\
\hline
\end{tabular}

Fig. 4. Packet format in the T-MPLS network

Each T-MPLS enabled switch in the network is implemented with a label table as shown in Table 1. When a specific flow based traffic policy associated with the flow ID is pushed from the PD-FE, the PE-FE (policy enforcement function entity) in the ingress node pushes a dynamic inner label for each incoming packet within this flow and distributes the labels as well as the policy information along the tunnel. An outer label is also pushed afterward, which is the designator to a LSP with the specific service class. As described above, in the case of TMPLS based metro transport network, the packet inherited the traditional MPLS format, but the outer labels used as indicating the MPLS tunnels (LSP) are configured by the management system. This approach is somehow similar with the IEEE 802.1ah defined PBT (Provider Backbone Transport) that two VLAN IDs are taken to identify one traffic flow for the purpose of QoS transport and traffic policy.

In the switching devices along the LSPs, the traffic flows will be forwarded and treated differently according to their outer label. When it comes to the egress node, the outer label is popped and the router policies the traffic flow according to its inner label. The usage of the double labels can be concluded as following: 
- Packet forwarding according to the outer label.

- Traffic policing according to the inner label.

Table 1 QoS mapping from network to transport level

\begin{tabular}{|l|l|l|}
\hline Indicator & Label & Usage \\
\hline User/Service ID & Outer label & $\begin{array}{l}\text { LSP /Traffic service } \\
\text { class }\end{array}$ \\
\hline Flow ID & Inner label & $\begin{array}{l}\text { Indicating the specific } \\
\text { traffic flow for the } \\
\text { ingress and egress } \\
\text { nodes policing }\end{array}$ \\
\hline
\end{tabular}

\section{MODELLING AND SIMULATION}

To illustrate the feasibility and possibility in realizing the Carrier Ethernet based on NGN, a Carrier Ethernet model implemented by OPNET (Fig. 5) is built here. Inside the model two scenarios are built: the admission control functions in the control plane with or without CAC function. The collected traffic statistics (end-to-end delay) and the comparison can be seen as a demonstration to the control plane influence to the transport network.

All the technologies and the concept described above are simulated into this model, including the separation of the control and transfer functions, the T-MPLS QoS transport functions (traffic policing and queuing), the QoS signaling and mapping from service level to the transport level and the call and admission control (CAC) inside the control plane.

All the nodes including the network ingress node, switches, area border router and the control plane (RACF) here are implemented with technologies described above. The end users running the VoIP, VoD and HTTP applications are placed in the access part of the transport network behind the ingress switch with the computer icon. The ingress node is the access node or can be treated as the DSLAM between the access network and the transport network. After the ingress node, there are the T-MPLS enabled switches, which form the T-MPLS metro network. The traffic is aggregated and switched through these switches. The nodes named "RACF" and "service center" reside at the other end of the transport network which can be seen as the core network. Node RACF plays the same role as the RACF in NGN. The node "service center" is the aggregation of the P-CSCF proxy and the RTSP proxy serving the SIP, RTSP (Real Time Streaming Protocol) and signaling.

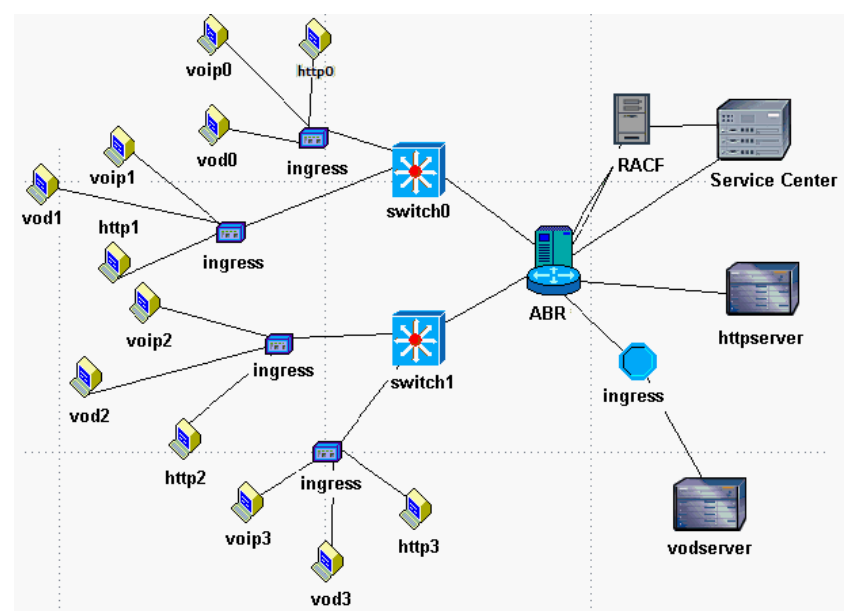

Fig. 5. OPNET model for Carrier Ethernet with control plane

Fig. 6 is the end-to-end delay of the scenario without CAC. The VoD application is set to send the data exceed the network resource reservation. From the figure we can see, as long as the transport policy gateway (token bucket) is working, the whole network is not totally corrupted with the "criminal bandwidth abusing". However, the two "hills" in the VoD packets delay shows the instability in the network without CAC. The network transport environment could be easily crashed by some "illegal" behavior such as trying to occupy a big amount of bandwidth.

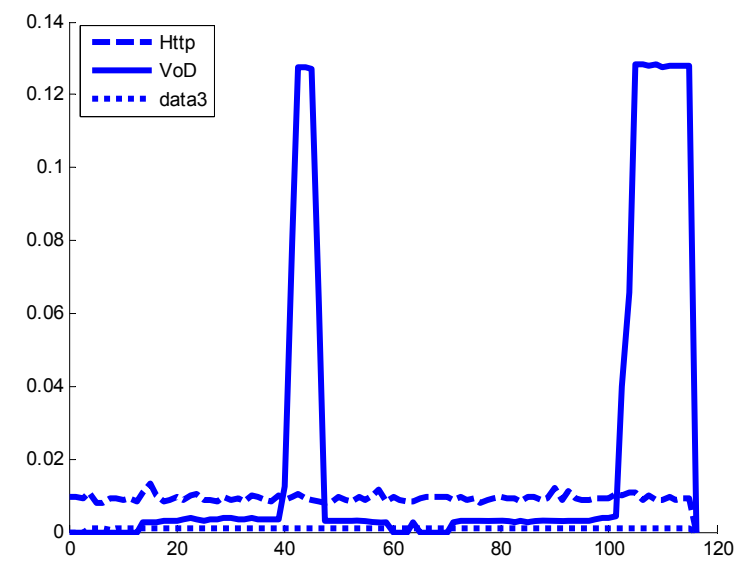

Fig. 6. End to end delay without CAC

However, under the simulating same conditions as the prior scenario, the situation will be different in the network with CAC function. Fig. 7 shows the simulation results for the transport network with CAC. 


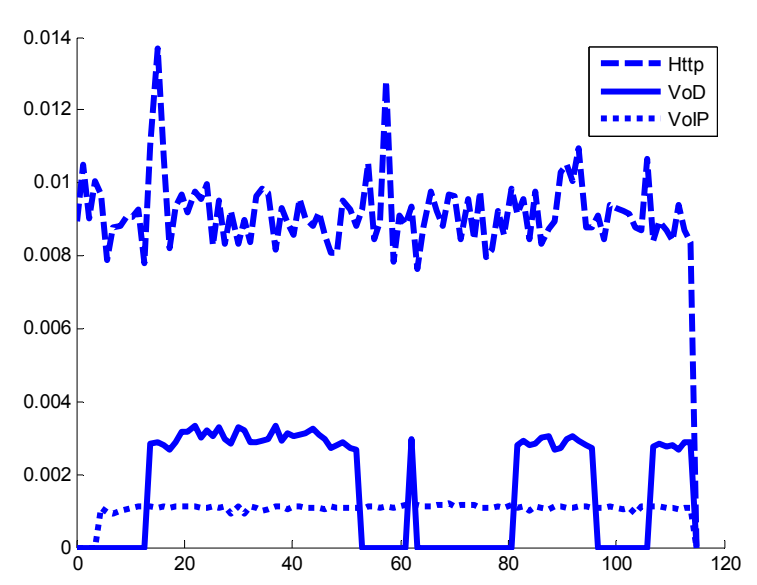

Fig. 7. End to end delay with CAC

The lags in the vod delay plot imply rejecting periods when there is not sufficient resource decided by the TRC-FE. Until the network gets the enough resource for the vod application, the following requests will be granted which is shown as the latter part of the red plot in Fig. 7. Additionally, the three plots in both the two scenarios also imply the different QoS service priority in the three applications. The VoIP has the highest priority while the Http has the lowest.

\section{CONCLUSION}

The paper work and the simulation case study demonstrate the combination of the Carrier Ethernet with the control plane in the NGN is a scalable and future proof solution. The Next Generation Network dedicates on providing a highly optimized functionality division, a set of open defined reference models, and more important an independent service and transport architecture. The attributes of Carrier Ethernet can be seen as a set of criterions for the transport network based on NGN, which the network architecture designers could refer to. On the other hand, the realization of the carrier class network transport services desires a unified and standard control mechanism from the NGN proposal.

Specifically, this paper contributes on building a typical Carrier Ethernet transport service solution by using $\mathrm{T}$ MPLS and illustrates the resource and admission control working schemes in the TMPLS based transport network. The control functions for the T-MPLS are proposed to be formulated into this independent control plane RACF on top of the transport network. The double label scheme in TMPLS label switching and flow based policy pushing mechanism are two fundamental methods to realize call and admission control (CAC) for the quality ensured transport. All in all, T-MPLS as a second layer based transport technology without routing procedures ensures the network scalability and the flexibility. The cooperation between TMPLS and control plane RACF based on the NGN makes T-MPLS fulfil the requirements desired by the carrier class Ethernet transport service.
Moreover, the simulation work demonstrates the efficiency and the feasibility of this Carrier Ethernet network with NGN control plane.

\section{REFERENCES}

[1] ITU-T Rec. Y.2001 "Next Generation Network Frameworks and functional architecture models, General overview of NGN" December 2004.

[2] ITU-T Rec. Y.2011 "General principles and general reference model for Next Generation Network" October 2004

[3] ITU-T Rec. Y.2111 "Resource and admission control functions in Next Generation Networks" September 2004 